\title{
Modification of the deoxyribose test to detect strong iron binding
}

\author{
Izabela Sadowska-Bartosz ${ }^{1 凶}$, Sabina Galiniak¹ and Grzegorz Bartosz ${ }^{1,2}$ \\ 1Department of Biochemistry and Cell Biology, Faculty of Biology and Agriculture, University of Rzeszów, Rzeszów, Poland; 2Department of \\ Molecular Biophysics, Faculty of Biology and Environmental Protection, University of Łódź, Łódź, Poland
}

\begin{abstract}
Deoxyribose test has been widely used for determination of reactivities of various compounds for the hydrox$\mathrm{yl}$ radical. The test is based on the formation of hydroxyl radical by $\mathrm{Fe}^{2+}$ complex in the Fenton reaction. We propose a modification of the deoxyribose test to detect strong iron binding, inhibiting participation of $\mathrm{Fe}^{2+}$ in the Fenton reaction, on the basis of examination of concentration dependence of deoxyribose degradation on $\mathrm{Fe}^{2+}$ concentration, at a constant concentration of a chelating agent.
\end{abstract}

Key words: chelation, deoxyribose test, desferrioxamine, DETAPA, EDTA, Fenton reaction, hydrogen peroxide, hydroxyl radical, iron, superoxide

Received: 03 July, 2016; revised: 31 July, 2016; accepted: 01 August, 2016; available on-line: 16 December, 2016

e-mail: isadowska@poczta.fm

Abbreviations: EDTA, ethylenediaminetetraacetic acid; DETAPA, diethylenetriaminepentaacetic acid; TBA, thiobarbituric acid; TCA trichloroacetic acid; TEMPO, 2,2,6,6-tetramethylpiperidine-1-oxyl

\section{INTRODUCTION}

The deoxyribose test is based on the use of a simple system, in which an iron complex reacts with hydrogen peroxide in the presence of ascorbic acid, presumably forming hydroxyl radicals. Hydroxyl radicals attack deoxyribose forming products that, upon heating with thiobarbituric acid (TBA) at low $\mathrm{pH}$, yield a pink chromogen. The test has been proposed to determine the rate constants of reactions of various compounds with the hydroxyl radicals since hydroxyl radical scavengers compete with deoxyribose for the hydroxyl radicals and diminish chromogen formation. A rate constant for reaction of the scavenger with hydroxyl radical can be deduced from the extent of inhibition of color formation. It is suggested that the deoxyribose assay is a simple and cheap alternative to pulse radiolysis for determination of rate constants for reaction of most biological molecules with hydroxyl radicals (Halliwell \& Gutteridge, 1981; Gutteride \& Halliwell, 1982; Halliwell et al., 1987). Reactivities of various substances for the hydroxyl radical have been estimated using this test (Bhat et al., 2001; Lapenna et al., 2002; Manoj \& Aravindakumar, 2003). The deoxyribose test has also been used to evaluate antioxidant activities of compounds and extracts (De et al., 2008; Guedes et al., 2013; Mokdad-Bzeouich et al., 2015). The test has been widely used and modified, i. a. to detect both antioxidant and prooxidant properties of compounds (Chobot, 2010). The mechanism of the test has been the subject of further studies. They demonstrated, i. a., that $\mathrm{Fe}^{3+}$, product of the reaction, contributes to deoxyribose degradation (Genaro-Mattos et al., 2009), that in reactions of hydroxyl radicals with deoxyribose five different deoxyribose radicals are formed, only one of which is transformed into malondialdehyde-like products reactive with TBA and that relative activity of antioxidants depends on the rate constants of many secondary reactions of antioxidants (Rachmilovich-Calis et al., 2009).

Moreover, the very basic assumption, i. e. that hydroxyl radical is the oxidant formed in the system and responsible for the deoxyribose degradation, has been questioned by various researchers. Winterbourn (1991) suggested that deoxyribose may be oxidized by iron(IV) species formed from $\mathrm{H}_{2} \mathrm{O}_{2}$ and $\mathrm{Fe}^{2+}$, but concluded that "the system is too complex for definitive identification of the Fenton oxidant". Similarly, hydroxylation of terephtalic acid by $\mathrm{Fe}^{2+}$ was ascribed to "cryptohydroxyl radical" ( $\mathrm{Fe}^{2+} /$ buffer complex) rather than to hydroxyl radical by Saran and coworkers (2000). It has also been suggested that at concentration ratios of $\left[\mathrm{O}_{2}\right] /$ $\left[\mathrm{H}_{2} \mathrm{O}_{2}\right]>100$ (prevailing in almost all cell compartments), hydrogen peroxide contributes negligibly to biological free radical oxidations and a non-identified "Fe-O" complex outcompetes $\mathrm{H}_{2} \mathrm{O}_{2}$-dependent oxidation pathways (Qian \& Buettner, 1999).

The Fenton system used for the induction of deoxyribose degradation in the deoxyribose test consists of hydrogen peroxide, $\mathrm{Fe}^{2+}$ ions, ethylenediaminetetraacetic acid (EDTA) chelating ferrous and ferric ions and ascorbate needed to recycle ferric ions produced in the Fenton reaction. Thus, the protection against deoxyribose degradation may be due not only to scavenging of the oxidant formed in the system, but also to prevention of the Fenton reaction by strong chelation of iron preventing its participation in the Fenton reaction. In this study, we attempted to find conditions for identification of the second possibility, which should be useful to detect compounds capable of strong iron binding, preventing the participation of ferrous ion in the Fenton reaction.

\section{MATERIALS AND METHODS}

All the reagents were from Sigma-Aldrich (Poznań, Poland). In a simplified version of the test, the samples contained $50 \mathrm{mM}$ phosphate buffer, $5 \mathrm{mM}$ deoxyribose, $\mathrm{pH} 7.4,80 \mu \mathrm{M} \mathrm{FeCl} 2$ and variable amounts of the compounds tested, or $80 \mu \mathrm{M}$ of a compound tested and variable amounts of $\mathrm{Fe}^{2+}$. In a full version of the test, $100 \mu \mathrm{M}$ ascorbic acid and $1 \mathrm{mM}$ hydrogen peroxide were also present. The mixtures were incubated at $37^{\circ} \mathrm{C}$ for $1 \mathrm{~h}$, then mixed with $250 \mu \mathrm{l}$ of $2.8 \%$ trichloroacetic 


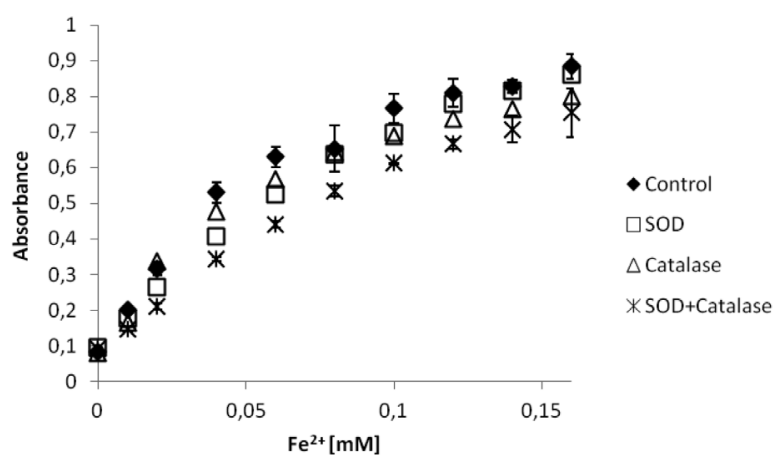

Figure 1. Effect of superoxide dismutase (SOD) and catalase and SOD+catalase on the deoxyribose degradation by $80 \mu \mathrm{M} \mathrm{Fe}{ }^{2+}$ in $50 \mathrm{mM}$ phosphate buffer, $\mathrm{pH} 7.4$.

Enzyme concentrations: $10 \mu \mathrm{g} / \mathrm{ml}$. For better transparency, S.D. is shown only for the extreme plots.

acid (TCA) and $250 \mu \mathrm{l}$ of $1 \%$ TBA (prepared in $0.1 \mathrm{M}$ $\mathrm{NaOH}$ ) and heated for $10 \mathrm{~min}$ at $100^{\circ} \mathrm{C}$. After cooling to room temperature, absorbance of the samples was measured at $532 \mathrm{~nm}$.

\section{RESULTS AND DISCUSSION}

$\mathrm{Fe}^{2+}$ induced deoxyribose degradation in the absence of chelators, both in the simplified and in the full version of the test. Deoxyribose degradation by $\mathrm{Fe}^{2+}$ in the simplified version of the test can be explained by a simple assumption of autoxidation of $\mathrm{Fe}^{2+}$ leading to formation of superoxide and, by its decomposition, hydrogen peroxide. Reaction of hydrogen peroxide with non-reacted $\mathrm{Fe}^{2+}$ would produce hydroxyl radical (Eqn. 1-3).

$\mathrm{Fe}^{2+}+\mathrm{O}_{2} \leftrightarrow \mathrm{Fe}^{3+}+\mathrm{O}_{2} \bullet$

$\mathrm{O}_{2} \bullet+\mathrm{O}_{2} \bullet+2 \mathrm{H}+\leftrightarrow \mathrm{O}_{2}+\mathrm{H}_{2} \mathrm{O}_{2}$

$\mathrm{H}_{2} \mathrm{O}_{2}+\mathrm{Fe}^{2+} \leftrightarrow \cdot \mathrm{OH}+\mathrm{HO}^{-}+\mathrm{Fe}^{3+}$

If this simple scheme was true, superoxide dismutase (SOD) producing hydrogen peroxide (reaction 2) and catalase dismutating it to $\mathrm{O}_{2}+\mathrm{H}_{2} \mathrm{O}$ should increase and decrease, respectively, the deoxyribose degradation. Alternatively, SOD could decrease the rate of reaction if superoxide is able to reduce $\mathrm{Fe}^{3+}$ formed. Experimental results do not conform with these predictions as SOD did not affect the degradation, catalase slightly decreased it and SOD+catalase produced a definite but small decrease of the extent of degradation (Fig. 1). It can be

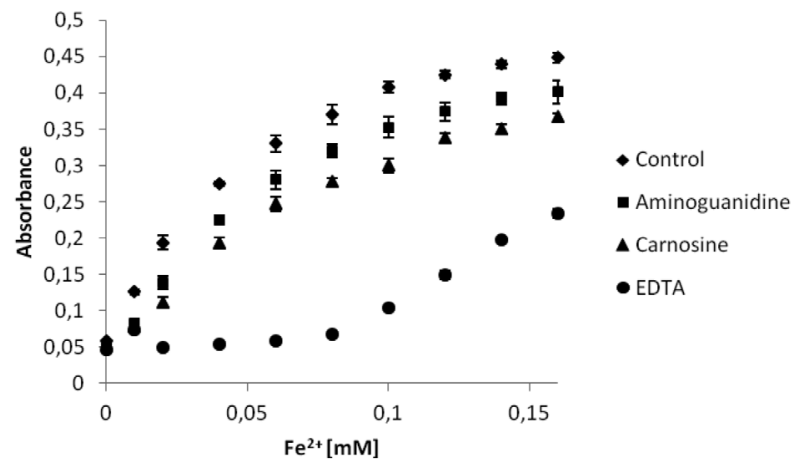

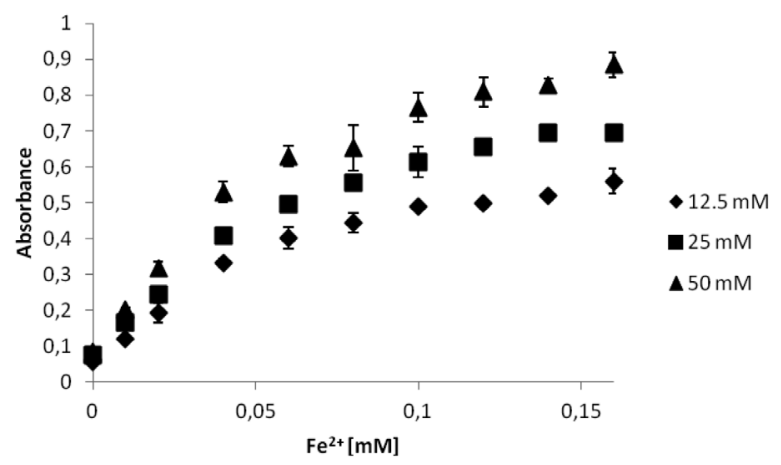

Figure 2. Effect of phosphate concentration $(12.5,25$ and 50 $\mathrm{mM}$ ) on the deoxyribose degradation by $80 \mu \mathrm{M} \mathrm{Fe} \mathrm{F}^{2+}$ in $50 \mathrm{mM}$ phosphate buffer, pH 7.4.

concluded that reactions in the system are more complicated than those presented by Equations (1)-(3); perhaps there is a significant contribution of direct degradation of deoxyribose by $\mathrm{Fe}^{3+}$ (Genaro-Mattos et al., 2009).

We studied the effect of phosphate concentration on the extent of deoxyribose degradation and found it to increase with the phosphate concentration (Fig. 2). Apparently, weak chelation of $\mathrm{Fe}^{2+}$ by phosphate increases the activity of ferrous ions. We checked various modifications of the test to distinguish between compounds binding weakly or not binding ferrous ions and those strongly binding these ions. Testing concentration dependence of deoxyribose degradation using constant $\mathrm{Fe}^{2+}$ concentration and variable concentrations of potential chelators was not sufficiently discriminative (not shown). However, examination of the dependence of the extent of degradation on the $\mathrm{Fe}^{2+}$ concentration at a constant $(80 \mu \mathrm{M})$ concentration of a potential chelator yielded two types of dependencies. In the absence of any chelator, a hyperbolic dependence was observed (Fig. 3, control). The same type of dependence was found for compounds known not to bind iron tightly (aminoguanidine, citrate, carnosine and rutin). In contrast, diethylenetriaminepentaacetic acid (DETAPA) and EDTA produced a concave plot demonstrating that until reaching a 1:1 stoichiometry, i. e. saturation of the binding capacity of the compound tested, the presence of the chelator decreased the participation of $\mathrm{Fe}^{2+}$ in the Fenton reaction (Fig. 3). The hyperbolic-type dependence was also found for 4-amino-TEMPO, captopril, carnosine, 4-cyano-1-hydroxycinnamic acid, cysteamine, ellagic acid, ferulic acid, gallic acid, genistein, 1-hydroxycinnamic acid, 4-hydroxyTEMPO, kempferol, metformin, naringin, propyl gal-

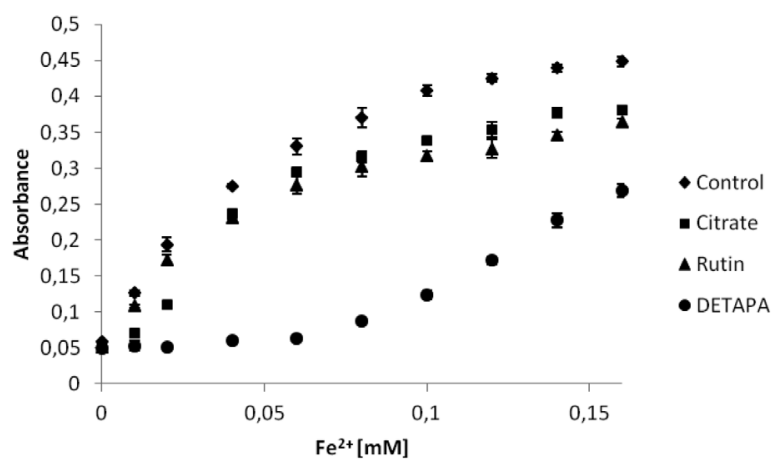

Figure 3. Dependence of deoxyribose degradation on the concentration of $\mathrm{Fe}^{2+}$ for various potential chelators in a simplified detection system. Concentration of potential chelators: $80 \mu \mathrm{M}$. 


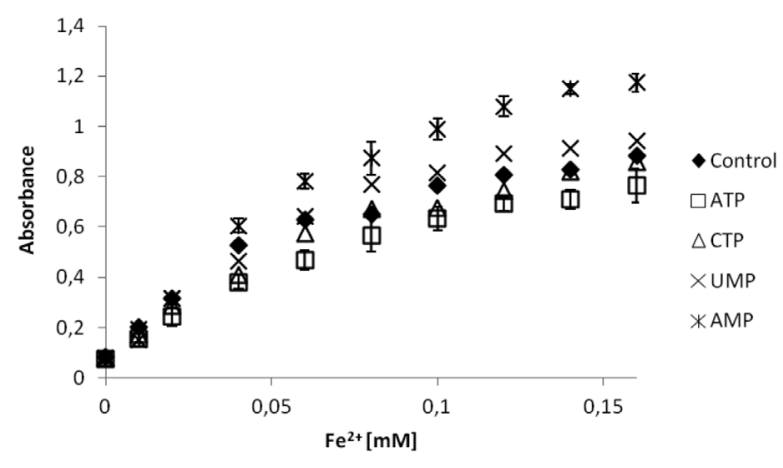

Figure 4. Dependence of deoxyribose degradation on the concentration of $\mathrm{Fe}^{2+}$ for nucleoside phosphates potential chelators in a simplified detection system.

For better transparency, S.D. is shown only for the extreme plots.

late, pyridoxine quercitrin, quinic acid, rutin, spermidine, TEMPO and tiron, while desferrioxamine and o-phenanthroline yielded a concave-type plot.

Our results indicate that the flavonoids tested, considered to be relatively strong iron chelators, do not bind $\mathrm{Fe}^{2+}$ strong enough to make the chelates unable to participate in the Fenton reaction. Similarly, nucleoside phosphates, considered to be relatively strong biological iron chelators, behaved like weak chelator in our test. Interestingly, white ATP and CTP decreased the extent of deoxyribose degradation, AMP and UMP increased it (Fig. 4). This property of nucleoside phosphates may be of biological relevance for iron-induced degradation in vivo. It could be suspected that chelators affect autoxidation of $\mathrm{Fe}^{2+}$ (reaction 1) rather than prevent participation of $\mathrm{Fe}^{2+}$ in the Fenton reaction (3). However, the same concentration dependence was observed in a full Fenton system, which is independent of $\mathrm{Fe}^{2+}$ autoxidation (Fig. 5).

Various tests have been proposed to evaluate binding of $\mathrm{Fe}^{2+}$ by pure substances, extracts and complex biological material including blood plasma, based mostly on spectral changes induced by the binding or on the inhibition of formation of $\mathrm{Fe}^{2+}$-ferrozine complex (Dinis et al., 1994; Khokhar \& Apenten, 2003; White \& Flashka, 1973). However, they do not allow for differentiation between compounds that are able or not to prevent participation of ferrous ions in the Fenton reaction, which is of considerable biological importance. The induction

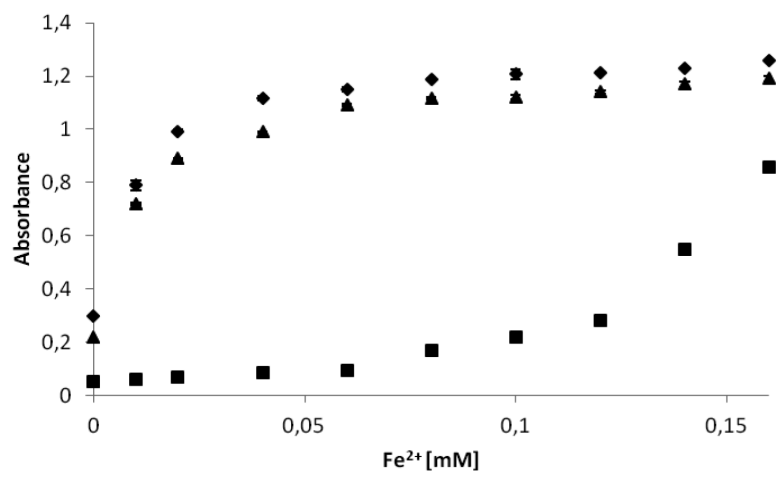

Figure 5. Dependence of deoxyribose degradation on the concentration of $\mathrm{Fe}^{2+}$ for various potential chelators in the full version of the test.

$(\diamond)$ Control; $(\boldsymbol{\Delta})$ Citrate, $(\boldsymbol{\square})$ DETAPA. Concentration of potential chelators: $80 \mu \mathrm{M}$. of deoxyribose degradation by iron salts and protective effects of various substances has been studied by Halliwell and Gutteridge (1981). This study pointed to a significant inhibition of the degradation by iron chelators (EDTA, DETAPA and desferrioxamine) and $\bullet \mathrm{OH}$ scavengers but did not point to the way of discrimination between both groups of substances. Such discrimination is difficult since virtually all substances, including iron chelators, scavenge $\bullet \mathrm{OH}$. In our opinion the modification of the deoxyribose assay proposed here, though qualitative only, may be useful in this respect and allow for identification of strong iron chelators among newly synthesized compounds.

\section{SIMPLE PROTOCOL TO DETECT STRONG IRON BINDING}

Reagents: (i) $50 \mathrm{mM}$ sodium phosphate buffer, $\mathrm{pH}$ 7,4; (ii) $20 \mathrm{mM}$ deoxyribose in (i); (iii) $1 \mathrm{mM} \mathrm{FeCl}_{2}$ in $1 \mathrm{mM} \mathrm{HCl}$ (prepare fresh before use); (iv) $1 \mathrm{mM}$ substance tested in (i) or another solvent, e. g. DMSO; (v) the solvent if different from (i); (vi) $2.8 \%$ of trichloroacetic acid (TCA); (vii) $1 \%$ thiobarbituric acid (TBA) in $50 \mathrm{mM} \mathrm{NaOH}$.

Procedure: Pipette $125 \mu \mathrm{l}$ of deoxyribose, (335-x) $\mu \mathrm{l}$ of buffer (i), $40 \mu$ of $1 \mathrm{mM}$ solution of the compound tested (iv) and increasing volumes (x) of $1 \mathrm{mM} \mathrm{Fe}^{2+}$ solution $(x=0,5,10,20,30,40,50,60,70$ and $80 \mu l)$ to successive Eppendorf tubes. Blank: $125 \mu \mathrm{l}$ of deoxyribose and $375 \mu \mathrm{l}$ of buffer (i). Incubate at $37^{\circ} \mathrm{C}$ for $1 \mathrm{~h}$. Then add $250 \mu \mathrm{l}$ of TCA solution (vi) and $250 \mu \mathrm{l}$ of TBA solution (vii). Heat at $100^{\circ} \mathrm{C}$ for $10 \mathrm{~min}$. Cool to room temperature, measure absorbance at $532 \mathrm{~nm}$ against a blank in a spectrophotometer or microplate reader. Plot absorbance vs $\mathrm{Fe}^{2+}$ concentration.

\section{Acknowledgements}

The study was performed within the COST CM1001 Action and supported by the NCN 2011/01/M/ NZ3/02065 and 2014/14/A/ST4/00640 grants.

\section{REFERENCES}

Bhat VB, Sridhar GR, Madyastha KM (2001) Efficient scavenging of hydroxyl radicals and inhibition of lipid peroxidation by novel analogues of 1,3,7-trimethyluric acid. Life Sci 70: 381-393

Chobot V (2010) Simultaneous detection of pro- and antioxidative effects in the variants of the deoxyribose degradation assay. I Agric Food Chem 58: 2088-2094 doi: 10.1021/jf902395k

De S, Adhikari S, Tilak-Jain J, Menon VP, Devasagayam TP (2008) Antioxidant activity of an aminothiazole compound: possible mechanisms. Chem Biol Interact 173: 215-223. doi: 10.1016/j. cbi.2008.03.011

Dinis TC, Maderia VM, Almeida LM (1994) Action of phenolic derivatives (acetaminophen, salicylate, and 5-aminosalicylate) as inhibitors of membrane lipid peroxidation and as peroxyl radical scavengers. Arch Biochem Biophys 315: 161-169

Genaro-Mattos TC, Dalvi LT, Oliveira RG, Ginani JS, Hermes-Lima M (2009) Reevaluation of the 2-deoxyribose assay for determination of free radical formation. Biochim Biophys Acta 1790: 1636-1642. doi: 10.1016/j.bbagen.2009.09.00

Guedes AC, Gião MS, Seabra R, Ferreira AC, Tamagnini P, MoradasFerreira P, Malcata FX (2013) Evaluation of the antioxidant activity of cell extracts from microalgae. Mar Drugs 11: 1256-1270. doi: $10.3390 / \mathrm{md} 11041256$

Gutteridge JM, Halliwell B (1982) The role of the superoxide and hydroxyl radicals in the degradation of DNA and deoxyribose induced by a copper-phenanthroline complex. Biochem Pharmacol 31: 2801-2805

Halliwell B, Gutteridge JM, Aruoma OI (1987) The deoxyribose method: a simple "test-tube" assay for determination of rate constants for reactions of hydroxyl radicals. Anal Biochem 165: 215-219 
Halliwell B, Gutteridge JM (1981) Formation of thiobarbituric-acid-reactive substance from deoxyribose in the presence of iron salts: the role of superoxide and hydroxyl radicals. FEBS Lett 128: 347-352

Khokhar S, Apenten RKO (2003) Iron binding characteristics of phenolic compounds: some tentative structure-activity relations. Food Chem 81: 133-140

Lapenna D, Ciofani G, Festi D, Neri M, Pierdomenico SD, Giamberardino MA, Cuccurullo F (2002) Antioxidant properties of ursodeoxycholic acid. Biochem Pharmacol 64: 1661-1667. doi:10.1016/ S0006-2952(02)01391-6

Manoj VM, Aravindakumar CT (2003) Reaction of hydroxyl radicals with $S$-nitrosothiols: determination of rate constants and end product analysis. Org Biomol Chem 1: 1171-1175.

Mokdad-Bzeouich I, Kilani-Jaziri S, Mustapha N, Bedoui A, Ghedira K, Chekir-Ghedira L (2015) Evaluation of the antimutagenic, antigenotoxic, and antioxidant activities of Eriobotrya japonica leaves. Pharm Biol 53: 1786-1794. doi: 10.3109/13880209.2015.1008145
Qian SY, Buettner GR (1999) Iron and dioxygen chemistry is an important route to initiation of biological free radical oxidations: an electron paramagnetic resonance spin trapping study. Free Radic Biol Med 26: 1447-1456

Rachmilovich-Calis S, Meyerstein N, Meyerstein D, Rachmilovich-Calis S, Meyerstein N, Meyerstein D (2009) A mechanistic study of the effects of antioxidants on the formation of malondialdehydelike products in the reaction of hydroxyl radicals with deoxyribose. Chemistry 15: 7717-7723. doi: 10.1002/chem.200802272

Saran M, Michel C, Stettmaier K, Bors W (2000) Arguments against the significance of the Fenton reaction contributing to signal pathways under in vivo conditions. Free Radic Res 33: 567-579

White JM, Flashka HA (1973) An automated procedure, with use of ferrozine, for assay of serum iron and total iron-binding capacity. Clin Chem 19: 526-528

Winterbourn C (1991) Factors that influence the deoxyribose oxidation assay for Fenton reaction products. Free Radic Biol Med 11: 353-360 